\title{
El aprendizaje de las metodologías de la enseñanza del inglés como lengua extranjera a través de preguntas, tareas y debates
}

\author{
CORAL IVY HUNT GÓMEZ \\ Universidad de Sevilla \\ Departamento de Didáctica de la Lengua \\ y la Literatura y Filologías Integradas \\ coralhuntg@us.es \\ ORCID.: https://orcid.org/0000-0001-6261-927X \\ D.O.I.: http://dx.doi.org/10.12795/JDU.2018.i01.49 \\ Pp.: 866-881
}

\section{Resumen}

Se realizó un segundo ciclo de mejora en la asignatura de último curso del Grado en Educación Primara: Métodos, Recursos Didácticos y Técnicas de Investigación en el Aula de Lengua Inglesa. Se dedicaron 10 sesiones teóricas y prácticas a implementar una metodología que se basa en el aprendizaje a través de preguntas sobre aspectos fundamentales de la materia, en tareas grupales para que los conocimientos teóricos se aplicaran y en un posterior debate conjunto con el fin de resolver posibles dudas y de afianzar conocimientos. El avance y la profundización de los participantes en el ciclo de mejora se midieron mediante la técnica de la escalera de aprendizaje a las respuestas 
iniciales y finales de 25 alumnos. Los resultados obtenidos han sido altamente positivos y, salvo excepciones, muestran una asimilación de contenidos y adquisición de destrezas y valores por parte de los futuros docentes.

Palabras clave: Métodos, Recursos Didácticos y Técnicas de Investigación en el Aula de Lengua Inglesa; Grado en Educación Primaria, Docencia Universitaria, experimentación docente universitaria, aprendizaje reflexivo.

\section{Breve descripción del contexto de intervención}

El ciclo de mejora se pone en práctica en el grupo de tarde de la asignatura "Métodos, Recursos Didácticos y Técnicas de Investigación en el Aula de Lengua Inglesa". Es una asignatura de cuarto curso del Grado en Educación Primaria, de 6 créditos ECTS, optativa aunque imprescindible para aquellos alumnos que quieran contar con un título de Grado en el que se incluya la Mención de Lengua Inglesa. En el grupo de tarde se imparte en tres clases de una duración de una hora y veinte minutos, en principio, dos clases teóricas y una práctica, lunes, martes y miércoles de 15:00 a 16:20. Una particularidad de la asignatura es que se imparte íntegramente en lengua inglesa. En el grupo hay 35 matriculados de los que asisten a clase de forma regular unos 25. El perfil del alumnado suele ser diverso y, en muchas ocasiones, el nivel de dominio de la lengua de comunicación en el aula no pasa de un B2, lo que dificulta el correcto desarrollo del proceso de enseñanza aprendizaje. A principio de curso se advierte a los estudiantes del nivel de lengua necesario para superar la asignatura, se realiza una prueba de nivel lingüístico y se les insta a que, como futuros profesores de lengua inglesa, adquieran un mejor dominio lingüístico. Los materiales están a disposición del alumnado en el espacio virtual. Este ciclo es el segundo que se realiza en la misma asignatura, 
ya que durante el curso 2017-2018 se implementó otro basado en el aprendizaje por preguntas, el trabajo autónomo y la inserción de la gamificación y las TIC en el aula con muy buenos resultados.

\section{Diseño del Ciclo de mejora}

Mapa de contenidos y principios básicos

El objetivo de la asignatura consiste en que los alumnos alcancen un determinado nivel de formulación en los contenidos. Algo que coincidiendo con la definición de García, Porlán y Navarro (2017, p. 56) se entiende como una aproximación provisional a un espacio del saber concreto. Al alcanzarse los objetivos que se establecen tendremos alumnos competentes, capaces de interactuar con el mundo a través de un conjunto de conceptos, procedimientos, valores y actitudes que les permiten actuar en contextos personales, sociales y profesionales (García, Porlán y Navarro, 2017, p. 57). A esta capacitación me gustaría añadir la académica.

A continuación se expone el mapa de contenidos de la asignatura engloba los conocimientos que se pretende que los alumnos alcancen, entre ellos están los conceptuales, los procedimentales y los actitudinales. La asignatura va dirigida a futuros maestros especialistas en Lengua Inglesa y se imparte en la lengua de trabajo por dos motivos: el primero de ellos es para fomentar la práctica entre los hablantes y el segundo consiste en familiarizar a los estudiantes con el class language, o la lengua propia del aula. Por último casi toda la literatura relevante se encuentra publicada en lengua inglesa.

El objetivo de la asignatura consiste en que los alumnos alcancen los conocimientos fundamentales en cuanto a los principales métodos de enseñanza del inglés como 
lengua extranjera: Grammar-Translation, Direct Method, Audiolingual, Reading Method, Silent Way, Suggestopedia, Community Language Learning, Total Physical Response, Communicative Language Teaching, Project-Based, Task-based. Estas metodologías se enseñan en clase en orden cronológico y se ven afectadas por el progreso de la Lingüística como ciencia y, por supuesto, por los cambios sociales que se producen. En la siguiente figura se expone de arriba abajo y cronológicamente, un mapa de los contenidos conceptuales teóricos con los que se espera que los alumnos se familiaricen.
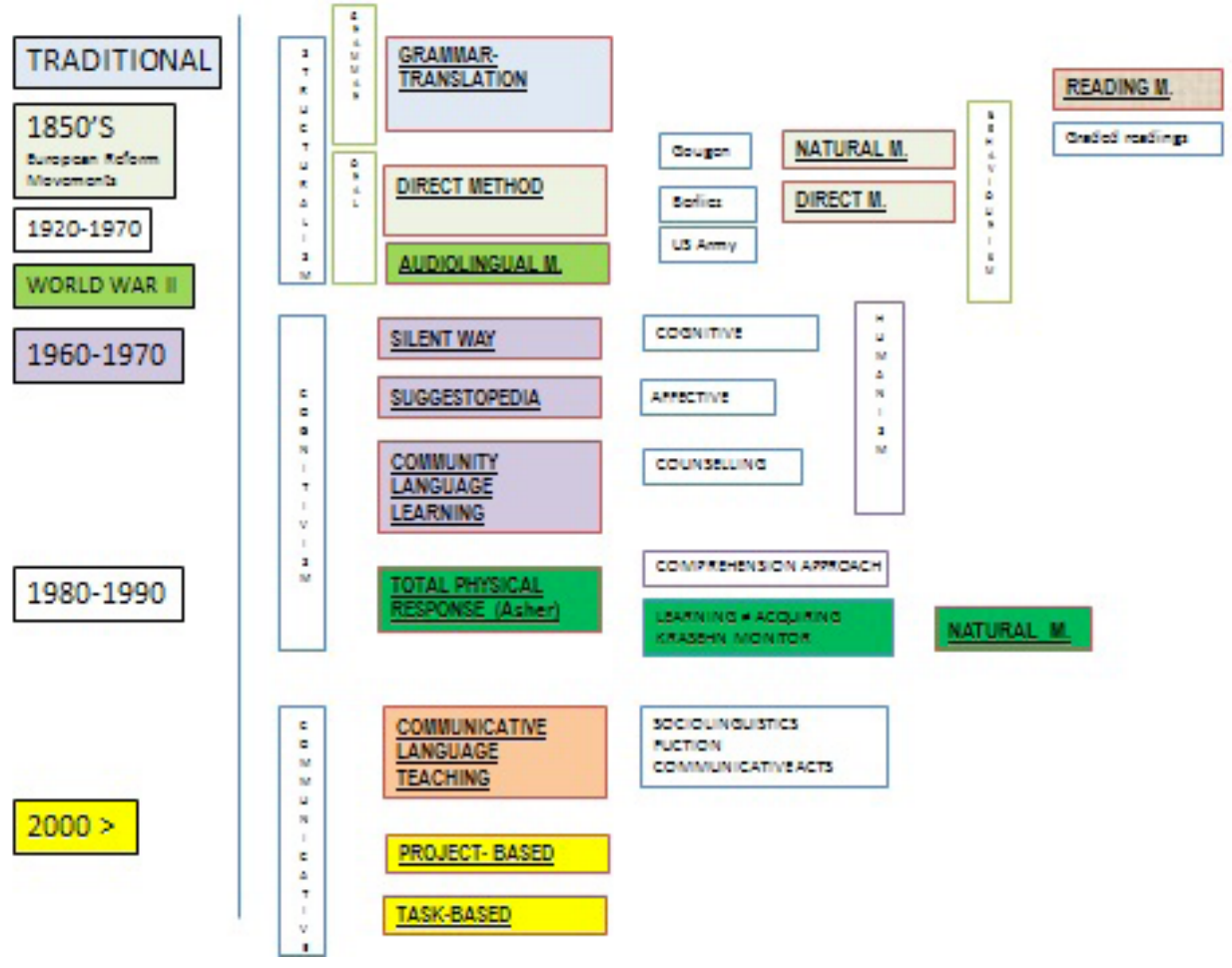

Conon:mevs CN ammonct

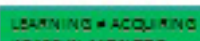

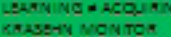
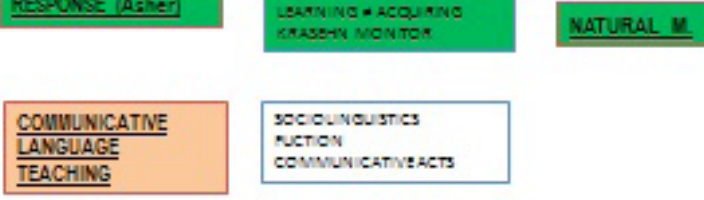

sec ou veustes

nuctan

COMNUN CATVIACTS

PROJECT-BASED

TASK-BASED

Figura 1. Mapa de contenidos conceptuales teóricos

Es interesante recalcar que, aunque las metodologías se suelan estudiar por separado las nuevas metodologías, en muchas ocasiones, se basan en metodologías cronológicamente anteriores o son el resultado de una reacción 
a la metodología previa. Por tanto, en la asignatura, los alumnos deben alcanzar una idea panorámica del efecto que pueden ejercer unas metodologías sobre otras (Figura 1).

Para poder comprender la evolución y la influencia de los paradigmas en la enseñanza de lenguas, los estudiantes deben asociar una serie de conceptos a cada una de las metodologías: el paradigma teórico en el que se desarrolla, papel del profesor, papel o interacciones entre alumnos y profesores, características del proceso de enseñanza, consideración hacia las emociones y los sentimientos, concepto de lengua y cultura, áreas destacadas, uso de la lengua nativa, evaluación, tratamiento del error, técnicas docentes. Todos estos conceptos ejercen una serie de interacciones muy marcadas entre ellos.

En relación a los contenidos procedimentales, los alumnos deben tener las capacidades de seleccionar y analizar materiales de enseñanza de lengua inglesa, de preparar las clases, de aplicar los métodos de manera práctica y también de habilidades de organización, síntesis y categorización. Por otro lado, los alumnos tendrán que alcanzar una serie de contenidos actitudinales: ser capaces de trabajar en equipo, optimizar los recursos a su alcance, respetar al alumnado y a los profesores, realizar exposiciones en público y valorar la excelencia en el desarrollo del trabajo.

Como se puede ver en la Figura 2, para poder alcanzar los objetivos de la asignatura y alcanzar las competencias necesarias para superarla, los alumnos deben desarrollar contenidos procedimentales y actitudinales. En cuanto a los contenidos procedimentales de análisis, síntesis, relación, organización y categorización, los alumnos los deben aplicar dentro de cada metodología para generar las relaciones entre los conceptos, pero también entre las metodologías de una manera panorámica. En este modelo también se incluyen las ideas de los estudiantes y algunos asuntos de carácter social o que afectan a otros ámbitos del conocimiento: concepto de lenguas y de culturas, 
prejuicios o ideas preconcebidas tanto de la lengua que estudian como de sus hablantes o de los conceptos que tienen que tratar, visión de los maestros.

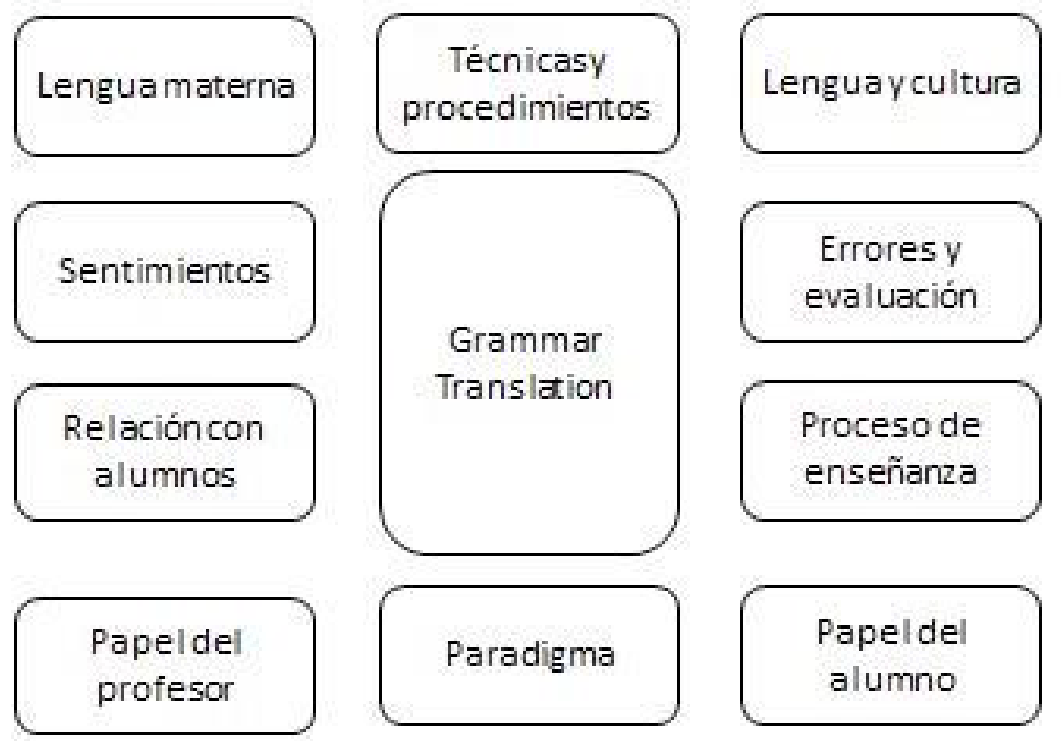

Figura 2. Contenidos conceptuales, procedimentales y actitudinales.

\section{Modelo metodológico y secuencia de actividades}

La clase se imparte íntegramente en inglés.

Se comienza con un pre-test sobre la temática de la sesión anterior para activar a los alumnos que se hace utilizando la herramienta de tele-voto Socrative. Así se evita tener que pasar lista, ya que la herramienta hace una lista de manera automática. Esto se hará en grupo y se irá mostrando una clasificación general en cada sesión.

Los alumnos trabajan en grupo y tienen a su disposición, desde la clase anterior, una serie de materiales y lecturas que versan sobre el aspecto en concreto que se va a trabajar en la sesión. Cada grupo tiene que realizar dos tareas.

Jornadas de Formación e Innovación Docente del Profesorado | № 1 (2018)

Esta obra se distribuye con la licencia Creative Commons 


\section{"La línea de los métodos".}

Consiste en hacer una línea imaginaria en la que se sitúe cada uno de los 10 métodos de enseñanza de la lengua inglesa que ya conocen, se denominará "La línea de los métodos". Para hacer el ejercicio más dinámico se puede hacer con una cuerda que se extiende en el suelo, se ponen dos tarjetas opuestas en cada uno de los extremos y cada grupo debe situar las tarjetas con los nombres de las metodologías según corresponda a lo largo de la cuerda en una posición más cercana a un extremo u otro (Figura 3)

La línea de los métodos

¿En qué medida se usa L1 en cada método?

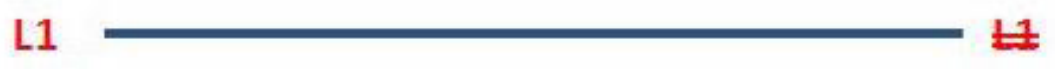

Figura 3. La línea de los métodos

\section{"Elaboración colectiva de respuestas y comunicación"}

Otra consiste en reflexionar y elaborar una respuesta conjunta a las preguntas que se plantean sobre un aspecto fundamental para la docencia en segundas lenguas en Educación Primaria: el uso de la lengua materna en el aula, las técnicas y procedimientos asociados a cada método, el concepto de lengua y cultura que se tiene, el modo de tratar los errores y la evaluación, el proceso de enseñanza que se lleva a cabo, el papel del alumno, el papel del profesor, las interacciones entre profesores y alumnos, el paradigma en el que se integra cada método y el papel que juegan los sentimientos en el aula.

Jornadas de Formación e Innovación Docente del Profesorado I № 1 (2018) Esta obra se distribuye con la licencia Creative Commons 
Para ello y para favorecer que todos los alumnos tengan la oportunidad de hablar en público, se nombra un portavoz de cada grupo y se hace un debate de la respuesta a las preguntas y se expone un resumen de los conceptos principales que se han explorado en el aula en base a las contestaciones de los alumnos. Por último, se informa a los alumnos del contenido de la próxima clase y se pone el material a su disposición en el espacio virtual. Una vez acabada la clase, se pone a disposición de los alumnos una breve explicación de las principales teorías que se llamará "Standing on the shoulders of giants" a la que tendrán acceso en el plataforma de enseñanza virtual. Los alumnos deberán leérsela o verla en el caso de que sean vídeos para repasar el contenido de clase de manera individual y así asegurarse de poder contestar correctamente al test del principio de la próxima clase.

\section{Secuencia de actividades}

A modo de ejemplo, a continuación se expone una tabla de la secuencia tipo de las actividades que se desarrollan en cada sesión. La sesión contiene una introducción en la que se repasan los contenidos previos, una parte de desarrollo en la que los alumnos deben realizar las dos tareas, una parte dedicada al debate de las conclusiones que se adquieran y por último se proporciona la información para la siguiente sesión. 
Tabla 1

Secuencia de aprendizaje

\begin{tabular}{|c|c|}
\hline Tiempo & Actividad \\
\hline $10^{\prime}$ & $\begin{array}{l}\text { Ejercicio de tele-voto basado en la sesión anterior } \\
\text { preguntas sobre la sesión anterior }\end{array}$ \\
\hline $40^{\prime}$ & $\begin{array}{l}\text { Planteamiento de las preguntas que deben resolver en grupo. } \\
\text { PROCESO DE ENSEÑANZA } \\
\text { ¿Cómo crees que se debería impartir el inglés en el } \\
\text { aula de educación primaria? } \\
\text { 2. ¿Crees que es necesario el contacto directo con la } \\
\text { lengua y su cultura (experiencia vivencial) o que } \\
\text { se puede aprender dentro del aula (perspectiva } \\
\text { cognosciva)? } \\
\text { 3Qué aspectos del currículum se deben destacar? ¿Qué } \\
\text { destrezas son más importantes : Reading, writing, } \\
\text { speaking, listsening? } \\
\text { Lecturas: } \\
\text { D’Aita, O. (2014).La metodología inductiva y el enfoque por } \\
\text { tareas para la enseñanza del presente en la asignatura de } \\
\text { inglés en primero de la Educación Secundaria Obligato- } \\
\text { ria del centro concertado Escola Virolai de Barcelona. Tra- } \\
\text { bajo fin de Máster. Universidad Internacional de la Rioja. } \\
\text { Págs. 10-14. } \\
\text { Larsen-Freeman, D. (2008) Techniques and Principles in } \\
\text { Language Learning. Oxford: Oxford University Press. } \\
\text { Martín Sánchez, M.A. (2010) Apuntes a la historia de la en- } \\
\text { señanza de lenguas extranjeras: la enseñanza de la gra- } \\
\text { mática. Approaches and Methods in Language Teaching. } \\
\text { 64-65. } \\
\text { Tejada Molina, G., Perez Cañado, M.L, Luque Agulló, G. } \\
\text { (2005) Current Approaches and teaching methods. Bilin- } \\
\text { guial Prommames En. McLaren, N., Madrid, D. y Bueno, A. } \\
\text { (eds.) (2005): TEFL in Secondary Education. Granada: Edi- } \\
\text { torial Universidad de Granada. }\end{array}$ \\
\hline
\end{tabular}

Jornadas de Formación e Innovación Docente del Profesorado | № 1 (2018)

Esta obra se distribuye con la licencia Creative Commons

Reconocimiento-NoComercial-SinObraDerivada

Internacional (CC BY-NC-ND 4.0.) 
Vídeos

- BRITISH COUNCIL. Teaching English

- https://www.teachingenglish.org.uk/article/ deductive-approach

- Deductive VS Inductive https://www.youtube.com/watch?v=g8rDOUpl-LC

¿Dónde sitúas cada método en la línea de los métodos?:

1. Grammar Translation,

2. Direct Method,

3. Audiolinguial method,

4. the Silent Way,

5. Dessugestopedia,

6. Community Language Learning,

7. Total Physical Response,

8. Communicative Language Teaching,

9. Content Based, Task Based \& Project Based Approaches.

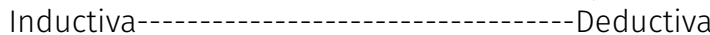

(conclusiones (conclusiones

por observaciones a partir de

que se transforman en las normas)

normas)

- Proceso de aprendizaje de idiomas (deductivo <> inductivo)

Debate sobre respuestas

$10^{\prime}$

Se indica el contenido de la próxima sesión y se ponen los materiales a disposición de los alumnos.

Standing on the shoulders of giants

\section{Cuestionario inicial y final para evaluar el progreso (Figura 6).}

Las siguientes preguntas, que se corresponde con el cuestionario inicial y final, tratan todos los aspectos principales de la asignatura y están diseñadas para que los alumnos interrelacionen los contenidos a través de la reflexión.

1. ¿Qué métodos de enseñanza de EFL conoces? Por favor, enuméralos y describe sus principales características. 
2. ¿Conoces algunos principios didácticos que puedas aplicar en el aula de Lengua Inglesa en tu futura vida profesional?

3. ¿Crees que es bueno usar la lengua materna en el aula de inglés?

4. ¿Qué importancia tiene cómo se tratan los errores en el aula?

5. ¿Qué incluirías en la evaluación de los alumnos?

6. ¿Cuál es el papel del profesor?

7. ¿Cuál es la mejor manera de aprender inglés?

8. ¿Qué deben hacer los alumnos en el aula y fuera de ella?

\section{Aplicación del ciclo de mejora}

\section{Relato resumido de las sesiones}

La clase previa al comienzo del ciclo se dedicó a explicar cómo se iba a desarrollar y a pedir a los estudiantes permiso para realizar el ciclo y para grabar las clases que lo componían. Al ser una intervención con el fin último de mejorar las prácticas docentes y del que, al ser una clase sobre metodologías didácticas, podrían sacar mucho provecho, se mostraron muy participativos. La primera clase se dedicó a que contestaran una serie de preguntas por escrito de acuerdo con sus conocimientos previos. Muchos alumnos se sorprendieron puesto que se les preguntó por cuestiones que no se habían tratado en el aula, sin embrago, al asegurarles que no tenía repercusión sobre la calificación de la asignatura y que servía para ver cuál era el punto de partida del estudiantado y así ver si se trataban con mayor o menor profundidad diversos aspectos, se relajaron y se pusieron a escribir. Algunos alumnos necesitaron terminarlo fuera del aula y lo entregaron más tarde.

Jornadas de Formación e Innovación Docente del Profesorado | № 1 (2018) Esta obra se distribuye con la licencia Creative Commons 
La primera sesión fue un poco confusa puesto que, a pesar de que se había explicado y de que el tiempo para cada tarea estaba claro, los alumnos tenían dudas en cuanto a las funciones y tareas de los miembros y no consiguieron terminar la tarea y contestar a las preguntas en el tiempo establecido. Esto hizo que la sesión se repitiera en la siguiente clase, ya con las tareas y la organización claras. Esto dio mejores resultados y rebajó la ansiedad de los estudiantes.

Los cuestionarios de Socrative fueron bien recibidos y los estudiantes se familiarizaron bastante rápido con la aplicación. Es un tipo de actividad que resulta muy adecuado para que el principio de la clase resulte activo y se repasen los conceptos.

La metodología de aprendizaje por tareas grupales, tener que establecer de en qué posición se colocan los distintos métodos con respecto a dos conceptos antagónicos de manera consensuada, al comienzo resultó complicado para los alumnos. No obstante, en las últimas sesiones, las interacciones y defensa de opiniones razonada entre ellos resultó fascinante y muy enriquecedora. A medida que avanzaba el ciclo, se acostumbraron a consultar las fuentes y a depender menos del profesor.

El debate del final de la clase, en muchos casos se quedaba algo corto de tiempo y los alumnos no desarrollaban conversaciones entre ellos y tenían posturas más bien uniformes. Otro aspecto que me gustaría destacar es que, durante el debate, la profesora intervenía modelando el conocimiento de los alumnos y volviéndolos a acercar al concepto de "respuesta única" que buscan. Esto quizás se debería matizar o hacer de otra manera en el futuro.

De acuerdo con las observaciones realizadas, la aplicación del ciclo de mejora ha resultado ser muy provechosa para los estudiantes en varios términos: mejora del trabajo en grupo, uso del inglés en el aula, razonamientos sobre contenidos, establecimiento de tareas y reparto de funciones, organización del tiempo y, por supuesto, mejora 
de los conocimientos teórico-prácticos relacionados con la asignatura.

\section{Evaluación del aprendizaje de los estudiantes}

En el Ciclo de Mejora Docente (CMD) se han utilizado tres sistemas de evaluación. El primero consiste en el análisis del test inicial y final de cada alumno para ver el progreso. Esto se hizo aplicando la técnica de las escaleras de aprendizajes. En total se analizaron los resultados de 8 preguntas y se plasmaron en gráficas como la que sigue:

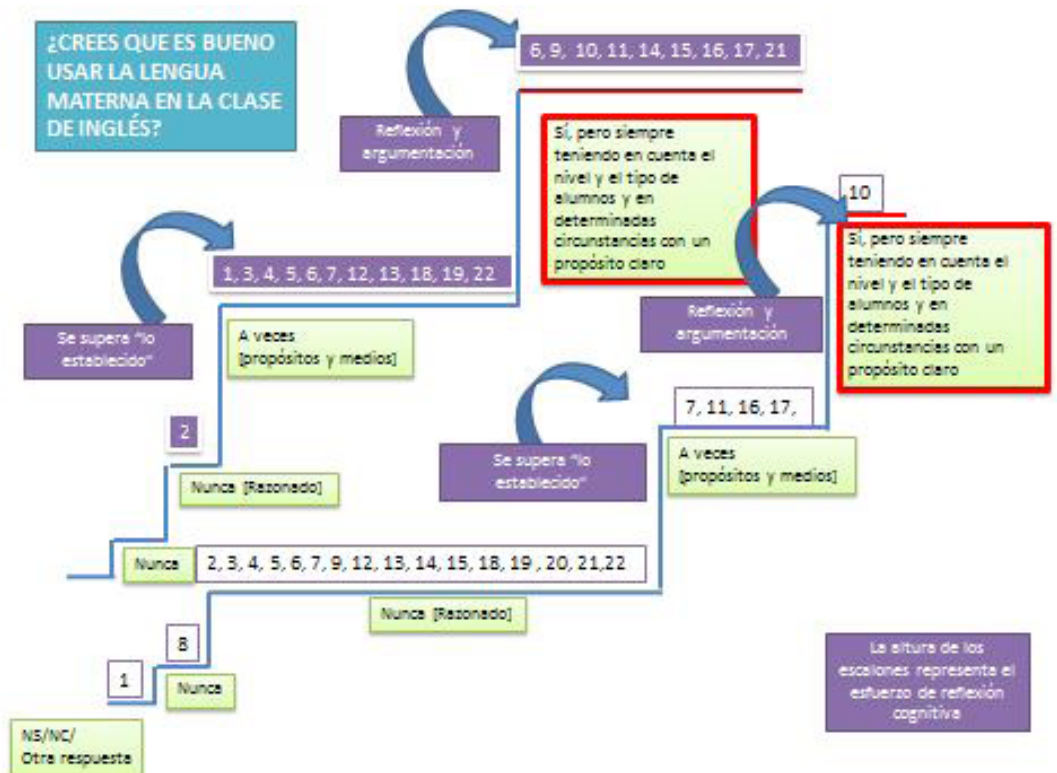

Figura 4. Escalera de aprendizaje inicial (abajo) y final (arriba)

El segundo método consistió en recoger las evaluaciones que proporciona el programa Socrative. Una vez creados los test de repaso para cada unidad, la propia aplicación genera una lista con los resultados colectivos e individuales con feedback para cada alumno. Esto resulta muy cómodo y productivo.

Jornadas de Formación e Innovación Docente del Profesorado | № 1 (2018)

Esta obra se distribuye con la licencia Creative Commons Reconocimiento-NoComercial-SinObraDerivada $\quad 4.0$ Internacional (CC BY-NC-ND 4.0.) 
Por último, como se recoge en el apartado anterior, se realizó un trabajo de observación directa en el aula por parte del Grupo de Formación e Investigación en Docencia Universitaria (FIDU). El CMD en su conjunto será estudiado y evaluado dentro del Programa de Formación e Innovación Docente del Profesorado de la Universidad de Sevilla.

\section{Evaluación del diseño puesto en práctica}

\section{Elementos que se mantienen y cambios necesarios}

Este CMD parte de otro que se realizó durante el curso 2017, que se insertaba en el curso de formación del profesorado y que obtuvo muy buenos resultados en general. Aunque el CMD ha resultado satisfactorio, algunos aspectos han funcionado mejor que otros.

Entre los elementos que se deben mejorar destacan las instrucciones que se dan a los alumnos, que deben ser más claras y el tiempo que se dedica a los debates al final de cada sesión, que ha resultado ser poco y demasiado orientado por la profesora. La observación de la profesora, aunque útil, no se ha realizado de manera sistemática. En el futuro estos aspectos deben mejorarse.

Los aspectos que se deben mantener puesto que sus resultados han sido muy buenos son: el uso de Socrative al comienzo de la clase y la combinación de las metodologías de aprendizaje por tareas y de aprendizaje por preguntas en grupo. Otro aspecto interesante y útil ha sido la evaluación del progreso por medio de la técnica de las escaleras de aprendizaje. 
En nuestra práctica docente se pretende seguir trabajando con un diseño metodológico innovador centrado en una metodología activa y que permita la reflexión y la puesta en práctica de los conocimientos teóricos. El trabajo en grupo y el consenso seguirán sirviendo como guía en el aula. Otro aspecto importante que se incorporará es el aprendizaje por tareas.

A partir de las experiencias y de lo aprendido en el CMD me gustaría incorporar los siguientes principios a mi docencia:

- El alumnado debe estar activado. La necesidad de concluir una tarea en un tiempo dado y de presentar resultados hace que los alumnos desarrollen sus capacidades y reflexionen durante la clase.

- Incorporación de una actividad breve de evaluación a través de la herramienta de televoto basada en la sesión anterior y que se refleje en la calificación.

- Las instrucciones que se proporcionen al alumnado deben ser claras y concisas. Deben estar organizadas de manera secuencial. Esto facilita la realización del aprendizaje por escrito.

- La realización de debates es muy productiva siempre que estén organizados y que los participantes dispongan de tiempo para que se desarrollen.

- La evaluación inicial de los alumnos debe realizarse con tiempo suficiente para ser analizada e incorporar los cambios necesarios a la docencia.

- La evaluación grupal e individual del conocimiento y destrezas adquiridas utilizando la escalera de aprendizaje es una herramienta muy valiosa.

- Los alumnos deben recibir feedback y conocer el estado de su progreso. 


\section{Referencias bibliográficas}

British Council (2017). Teaching English. Disponible en: $\quad$ https://www.teachingenglish.org.uk/article/ deductive-approach

D’Aita, O. (2014). La metódología inductiva y el enfoque por tareas para la enseñanza del presente en la asignatura de inglés en primero de la Educación Secundaria Obligatoria del centro concertado Escola Virolai de Barcelona. Trabajo fin de Máster. Universidad Internacional de la Rioja.

García Díaz, E, Porlán, R. y Navarro, E. (2017). Los fines y los contenidos de la enseñanza. En R. Porlán (Coord.), Enseñanza Universitaria. Cómo mejorarla (pp. 55-72). Madrid: Editorial Morata.

Larsen-Freeman, D. (2008). Techniques and Principles in Language Learning. Oxford: Oxford University Press.

Martín Sánchez, M.A. (2010). Apuntes a la historia de la enseñanza de lenguas extranjeras: la enseñanza de la gramática. Tejuelo, 8, 59-65.

Tejada Molina, G., Pérez Cañado, M.L, Luque Agulló, G. (2005). Current Approaches and teaching methods. Bilinguial Prommames. En N. McLaren, D. Madrid, y A. Bueno (Eds.), TEFL in Secondary Education (pp. 156-208.). Granada: Editorial Universidad de Granada.

Jornadas de Formación e Innovación Docente del Profesorado I № 1 (2018) Esta obra se distribuye con la licencia Creative Commons 